\title{
Screen use and its impact on teenagers: Ongoing education and open-minded vigilance
}

\section{HRONIQUE • Technologies en éducation}

\section{The stork effect: correlation or causality?}

The legend of the storks of Alsace dates from 1850, when people first conceived the notion that a stork on the wing meant a baby

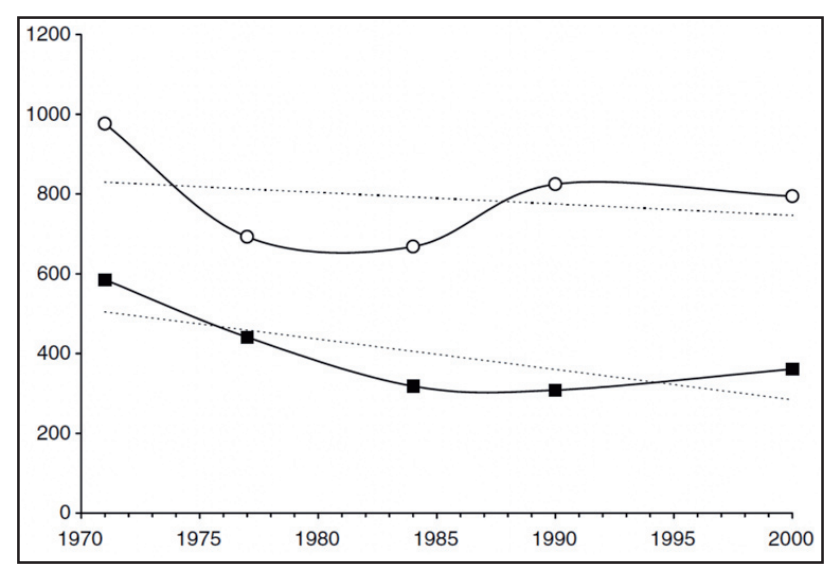
on the way. According to local folklore, the Kinderbrunnen, or "child-fountain," was a well of souls waiting to be reincarnated. A woman who longed to get pregnant leaned over the well and made a wish. An elf then fetched a baby from the underground depths. A further refinement to the story was that beneath Strasbourg Cathedral lay a subterranean lake, where the children's souls amused themselves before being born. The elf embarked on the lake in his silver boat, caught a baby with a golden thread, and deposited it on the edge of the well. A stork then delivered the

Figure 1

Correlation between births and stork nests. Höfer and colleagues (2004). baby to its cradle in the proud parents' home. This rather charming fantasy has travelled the world as far as East Asia. As it turns out, the story could actually be true, at least in one statistical sense, and in Germany. This is what Höfer and colleagues demonstrated in 2004 (Figure 1) in their article published in Scientifique Paediatric and Perinatal Epidemiology called "New Evidence for the Theory of the Stork." ${ }^{1}$ Several other authors provided similar proofs. To do so, they correlated the number of births in homes where storks were nesting on the chimney. In fact, the correlations were quite high, which might seem to confirm the myth that the babies arrived sound and healthy thanks to the storks. In reality, however, all the researchers used the fairy tale to illustrate the difference between a correlation and a cause-and-effect relationship. The highly significant correlations 
were explained as follows: in homes where newborns lived, the heating was turned up higher during the day. The storks greatly preferred to build their nests on the warmer chimneys, and they habitually built them in the daytime when the temperatures peaked. Therefore, despite the strongly positive statistical correlations, the arrival of the babies cannot be attributed to the arrival of the storks. The statistical results are explained instead by the storks' partiality for the more comfortable chimneys.

\section{A correlation is a statistical relationship that does not establish whether one variable acts on another. A causality is a relationship where one variable acts directly on another. Ergo, correlation is not the same as cause-and-effect, and it is important not to confuse the two, notably in the abundant literature on the impact of screen time on youth.}

So why bring up the storks at all? As a symbol of hope? No. My purpose is to clarify the immense difference between a significant correlation and a cause-and-effect relationship, whatever the significance. It is also one of the best known examples — and I use it liberally in my courses - to teach the difference between correlation and causality. Here is the essential difference between the two: a correlation is a statistical relationship that does not establish whether one variable acts on another, whereas a causality is a relationship in which one variable acts directly on another. So, just because two variables are correlated, one does not necessarily cause the other. Unfortunately, in the abundant literature on how screen time impacts youth, correlation and causality tend to be confused. In terms of pure numbers, looking at an analysis of 13,659 studies on this topic (Figure 2), ${ }^{2}$ it is tempting to conclude that TV 1) causes serious psychological problems such as depression, 2) leads to obesity, 3) diverts us from exercising, and (Figure 1. Figure presented by Höfer and his colleagues (2004) highlighting the correlation between storks and birth rates in Germany.) 4) automatically reduces our hours of sleep.

The first study to explore the issue of screen time was written in the 1970s, but it was in 2005 (392 publications in that year alone, and 11,457 publications since, for $83.9 \%$ of all published studies so far) that the research really took flight. In 2019, a record year, 1,370 studies were produced.

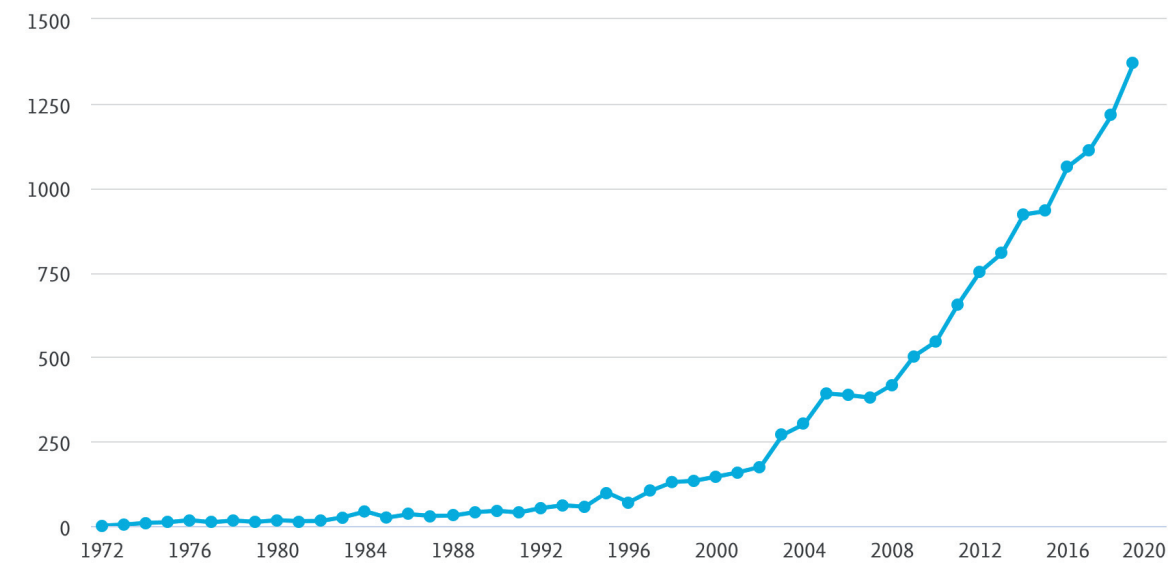

Figure 2

Growth in the number of articles on the impact of screen time published in scientific journals since 1972 $(n=13,659)$. 
Far too many of these studies concluded, based on correlations, that TV screen time wields four main negative impacts: more psychological problems, weight gain, disinterest in physical activity, and loss of sleep.

Based on correlations, studies have overwhelmingly concluded on four negative impacts: more psychological problems, weight gain, less physical activity, and loss of sleep.

Since 2005, the majority of studies have addressed health problems that are thought to be associated with screen time. Of these problems, $45.6 \%$ concern diverse psychological disorders (e.g., depression), $13.3 \%$ weight gain, $12.3 \%$ disinterest in physical activity, and $7.2 \%$ sleep loss. The correlations "prove" it, and they are generally quite significant. It's the storks and babies all over again. The only problem is that very few scientifically robust studies have sought to establish non-spurious cause-and-effect relationships.

The fact that two variables are correlated does not mean that one causes the other.

So, does owning a cell phone really make a teenager obese? Does watching Netflix make you lose your taste for sports? Does spending time on social media make you depressed? Does screen time make you snack at McDonald's instead of preparing a healthy meal? Yes, if we believe what the correlations say. However, we must never forget that although correlations are associations, they are not cause-endeffect relationships. A correlation between two variables does not mean that one causes the other. We must keep this essential distinction firmly in mind before leaping to conclusions.

What we know for sure is that teenagers spend a lot of time staring at screens. In a study by Common Sense Media in 2015, ${ }^{3}$ teenagers reported that they watched a screen for almost 9 hours a day, or about 3,276 hours a year. More recent studies suggest that even teenagers are starting to worry about this. ${ }^{4}$

\section{Main shortcomings of studies on the impact of screen time on youth}

In the literature on screen use by youth, six main shortcomings have been identified:

- First, and as illustrated by the stork effect, hasty conclusions are drawn all too often when correlations are confused with cause-and-effect relationships.

- Second, the studies contain certain methodological errors. For instance, important data may be missing, including the youth's socioeconomic status, which is liable to play a role in the health issues associated with screen use.

- Third, propaganda tends to rear its ugly head. For example, technology devices may be viewed as nothing less than diabolical. Tellingly, since 2005 , only $2.9 \%$ of studies have included any positive impacts of screen use. In other words, $97.1 \%$ of studies present only the negative impacts. Why this almost total refusal to look at the positive impacts? Especially given that many studies have demonstrated how technology can help students learn. A case in point is the exhaustive metaanalysis by Zeng and colleagues in $2016 .^{5}$ 
- Fourth, as youth continue to use ever more apps (e.g., Snapchat, Instagram, YouTube, Facebook, TikTok), which are developed ever more rapidly, it becomes ever more difficult to design experimental protocols to accurately determine the specific effects of each app. So the research tends to lag behind technology advances.

- Fifth, the recommendations that are offered to the general public may be premature. Researchers disagree on the ideal screen time: 15 minutes, half an hour, an hour? This advice is rarely based on the sound evidence of rigorous studies, and seems instead to be flavored according to personal taste. Why would any specific time be optimal? Why would less than 10 minutes a day be best for 12- to 15-year-olds? Don't they use screens at school? These inconsistent guidelines, which are often disconnected from the reality of youth, (sometimes called "ostrich head in the sand" views), tend to be unrealistic in practice.

- Sixth, and perhaps the most thoughtless shortcoming, is that what is displayed on the screen has been systematically ignored. Most studies consider screen time in a holistic manner, with little regard for the content. Are the kids playing Fortnite (an intensely popular game among adolescent boys) or Candy Crush (beloved by adolescent girls)? Would the impact be the same if they were reading Jane Austen's Pride and Prejudice on a tablet? No need to conduct a study to find the answer: it's inconceivable that the impact would be the same. The ways that youth use their screens should be a central research variable. Yet this is almost never the case, and data on this aspect are practically nonexistent.

\section{One thing is sure: teenagers spend lots of time staring at screens.}

\section{Smart phones and texting: do they intensify screen time problems?}

"Merry Christmas" was the very first text message (short messaging service-SMS) that Neil Papworth sent to Richard Jarvis on December 3,1992, almost three decades ago. Papworth, a British engineer, did not foresee that his invention would spread like wildfire. The movement began rather timidly, but in time gained so much momentum that it has transformed the way we communicate with each other. In 1995, the average American sent fewer than one text per month (estimated at 0.4). It was only in 1999 that users could exchange texts between networks. That's when the frenzy started. Texts were sent around the globe, and texting quickly became the predominant means of communication. In a massive societal shift, eight trillion text messages are now sent annually, and this does not include the messages sent via Snapchat, Instagram, WhatsApp, and Facebook Messenger. For the last 10 years, teenagers have been the most fervent texters. What can account for this? There are many reasons, but one that seems obvious is that they enjoy texting, an explanation that has been supported by numerous studies. ${ }^{6}$ Moreover, given the astronomical number of texts that they produce (Table 1), it is widely believed that teenagers would rather write to each other than converse. In 2010, they were sending 3,339 texts per month. ${ }^{7}$ Girls were quicker to jump on the bandwagon, with an average of 4,050 texts per month compared to 2,539 for boys. Today, the monthly average for teenagers is 6,200 texts, and the numbers continue to grow. 


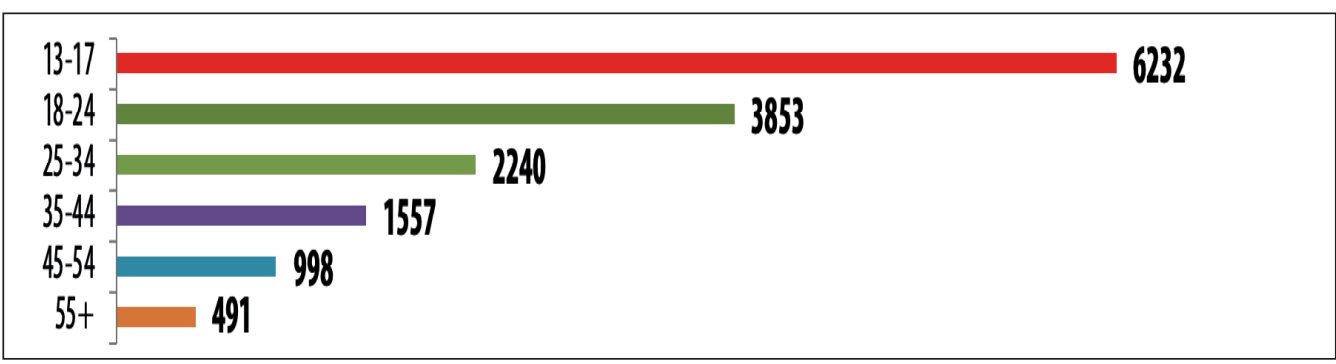

Table 1. Texts sent per month by age group ${ }^{8}$

But let's leave texting aside for the moment. The more pressing concern for public health pundits is the smart phone. This is the villain most often named in studies on the impact of screen time. It is estimated that over five billion people own a smart phone. ${ }^{9}$ That's almost three-quarters of the planet's entire population. Hence, many studies have striven to prove that smart phones, which provide teenagers with instant access to a screen, are accentuating the problem. Importantly, the proportion of teenagers with a smart phone is huge (89\%, according to recent statistics), and it will only increase, year after year. ${ }^{10}$

A buge proportion of teenagers owns a smart phone (89\%, according to recent statistics), and this will only increase, year after year.

\section{Conclusion}

Most societies want their young people to be healthy. This concern has taken many forms over the years, as in Canada and Québec. In the early 1970s, the Canadian government created a national non-profit organization called ParticipACTION ${ }^{11}$ in response to a 1969 study by the National Advisory Council for Fitness and Amateur Sport. The study concluded that the "future well-being of Canadians was in jeopardy" because Canadians were "largely sedentary (i.e., inactive), unfit, and, worse yet, uninterested in the concept of exercise and its health benefits."

Half a century on, the astonishing diffusion of smart phones among teenagers seems to support this dire prediction. Parents, teachers, and public health experts continue to wring their hands about sedentary lifestyles. Yet despite their fears, a review of the literature indicates that the impact of screen time on youth may not be so dreadful after all. In a recent (2019) critique of secondary analyses of large-scale social datasets (including 355,358 subjects) published in Nature: Human Behaviour, Orben and Przybylski ${ }^{12}$ point out that the effects of digital technology use on adolescent well-being are too small to be taken seriously.

Orben and Przybylski's recently (2019) showed that, despite the abundant research that has sought to determine the contrary, screen time and technology use has only a tiny effect on well-being.

At most, only $0.4 \%$ of the variation in well-being could be explained. Furthermore, only teenagers who were on their screens for more than 63 hours a month appeared to be aware of any negative impact on their well-being. The authors suggest applying more consistent analytical methods and a broader approach to examine the issue in future. At the same time, like most studies in the field, they failed to identify any benefits for teenagers of technology use. 
In light of the available evidence, we are forced to admit that we know very little about how screen time impacts the health and well-being of teenagers. Large-scale studies are needed in Québec and elsewhere to deepen our understanding of what has become a worldwide concern. But importantly, these studies should account for how teenagers use their screen time, including the positive uses.

\section{There must be some benefits of technology use, even for teenagers.}

It is not lack of data that will prevent us from determining what balanced screen use should look like (although it might be extremely hard to achieve such a balance in practice). A reasonable target for screen time should take into account the contemporary reality of teenagers, including the brisk pace of technology advances. These new advances also call for flexible guidelines and rules that can move with the times. Above all, we must not bury our heads in the sand.

Can we not stop demonizing digital screens? Amid the barrage of research on how they ruin our lives, let's take a minute to remember that these devices can actually help students learn. Fortunately, we are spoiled here in Québec, with its new digital education policy. In April 2019, the Québec government instituted a Digital Competency Framework. ${ }^{13}$ The aim is to "foster the development of digital competency throughout the education community so that Quebecers may be autonomous and exercise critical judgment in their use of digital technologies. Digital competency is essential in today's world, in which technological innovations are transforming the labour market and the skills that employers are looking for."

In sum, screen use, at home and at school, should be guided by judgment, discernment, enjoyment, and perhaps moderation. Parents, teachers, and public health experts should not be ruled by pessimism in the face of challenges, nor swept away by enthusiasm for the vast possibilities. Let's be clear-sighted, level-headed, and open-minded. We have to be ready to embrace new advances with a reasoned intelligence, and with a view to furthering education while preventing abuse. The French philosopher Michel Serres (1930-2019) in a recent book called Thumbelina (2014), ${ }^{14}$ describes a new human that is being born through new technologies (e.g., schoolgirls texting their friends). He argues that we are witnessing just the latest in a lengthy series of cultural revolutions based on technology, including the printing press, the telephone, and the television. Serres consistently advocated respectful dialogue between all parties. Can we not have a respectful dialogue with our teenagers so that they can derive the full benefits from their screens?

He or she writes differently. After watching them, with admiration, send an SMS more quickly than I could ever do with my clumsy fingers, I have named them, with as much tenderness as a grandfather can express, Thumbelina (Petite Poucette) and Tom Thumb (Petit Poucet). ${ }^{15}$

\section{Notes}

Höfer, T., Przyrembel, H. \& Verleger, S. (2004). New evidence for the theory of the stork. Paediatric and Perinatal Epidemiology, 18(1), 88-92.

2 We conducted a systematic analysis of health, psychology, and social sciences studies in the CINAHL, ERIC, MEDLINE, PsycINF0, Scholar, and Scopus databases. A thematic analysis of the retrieved references identified further main study conclusions. Although the review covered years 2018 and 2019 only due to time constraints, most studies also cited previous studies.

3 https://www.commonsensemedia.org/about-us/news/press-releases/landmark-report-us-teens-use-an-average-of-nine-hours-of-media-per-day 
(1/www.pewresearch.org/internet/2018/08/22/how-teens-and-parents-navigate-screen-time-and-device-distractions/

Zheng, B., Warschauer, M., Lin, C.-H. \& Chang, C. (2016). Learning in one-to-one laptop environments: A meta-analysis and research synthesis. Review of Educational Research, 86(4), 1052-1084.

6 Bruehlman-Senecal, E., Aguilera, A. \& Schueller, S. M. (2017). Mobile phone-based mood ratings prospectively predict psychotherapy attendance. Behavior Therapy, 48(5), 614-623.

7 Source: http://www.nielsen.com/us/en/insights/news/2010/u-s-teen-mobile-report-calling-yesterday-texting-today-using-apps-tomorrow.html

8 Table constituted from seven sources: Pew Research Center, Experian, Cappex, Twilio, Teckst, Portio Research, and Text Request. http://www.pewinternet.org/2015/04/09/teens-social-media-technology-2015/, http://www.experian.com/marketing-services/webinars

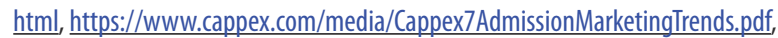

https://www.twilio.com/learn/commerce-communications/how-consumers-use-messaging, https://teckst.com/19-text-messaging-stats-that-will-blow-your-mind/, http://www.portioresearch.com/en/blog/2013/17-incredible-facts-about-mobile-messaging-that-you-should-know.aspx, https://www.textrequest.com/blog/many-texts-people-send-per-day/, https://www.textrequest.com/blog/texting-statistics-answer-questions/ https://doi.org/10.1038/s41562-018-0506-1

13 http://www.education.gouv.qc.ca/en/current-initiatives/digital-action-plan/digital-competency-framework/

14 Serres, M. (2012). Thumbelina. The Culture and Technology of Millennials. Translated by Daniel W. Smith. Rowan \& Littlefield International.

15 Serres, M. (2014). Thumbelina. The Culture and Technology of Millennials. Translated by Daniel W. Smith. Rowan \& Littlefield International. https://www.rowmaninternational.com/book/thumbelina/3-156-bbff13ae-9e2b-4e9b-a735-8ec8756de17d

\section{Pour citer cet article}

Karsenti, T. (2019). Screen use and its impact on teenagers: Ongoing education and open-minded vigilance. Formation et profession. 27(3), $146-152$. http://dx.doi.org/10.18162/fp.2019.a191 